\title{
Optimal Auctions for Asymmetrically Budget Constrained Bidders*
}

\author{
Alexey Malakhov and Rakesh V. Vohra \\ Department of Finance, Kenan-Flagler School of Business \\ University of North Carolina \\ Chapel Hill, NC 27599 \\ Department of Managerial Economics and Decision Sciences \\ Kellogg School of Management \\ Northwestern University \\ Evanston IL 60208, USA
}

December 2005

\begin{abstract}
We consider an environment with a single divisible good and two bidders. The valuations of the bidders are private information but one bidder has a commonly known budget constraint. For this environment we derive the revenue maximizing subsidy free incentive compatible auction. We also examine the case when the budget constraint is private information but bidders must post a bond.
\end{abstract}

${ }^{*}$ The research was supported in part by the NSF grant ITR IIS-0121678. 


\section{Introduction}

A seller facing a collection of budget constrained bidders recognizes that such constraints limit the revenue she can generate from the sale of an item. The most obvious limitation is the size of the budgets themselves. They also hinder competition. If one knows that a competitor cannot bid beyond a certain amount, this reduces the bid one needs to place to secure the object. To stimulate competition amongst the bidders, the seller must favor some bidders and handicap others, perhaps by setting reserves or subsidies. While such schemes may increase expected revenues they do so by imposing greater risks on the seller. A reserve, for example, would mean that a trade is sometimes not executed. Finding the right combination of carrots and sticks in this environment to stimulate bidding is therefore an important problem. It is also a difficult one because an analysis must deal with the uncertainty in both a bidders valuations and her budget constraint. The difficulties are not unique to this instance but arise in many environments where the private information of a bidder is multi-dimensional (see, for example, Rochet and Chone (1998) and Rochet and Stole (2003). For this reason attention has focused on special cases.

Laffont and Robert (1996) assume that valuations are private but that all bidders have the same common knowledge budget constraint. In this environment they derive the subsidy free revenue maximizing Bayesian incentive compatible auction. Since all bidders have the same budget constraint, the analysis sheds no light on the balance between carrots and sticks. The same environment is assumed in Maskin (2000) where he examines constrained efficiency. Again, the symmetry in the budget constraints sheds no light on how the seller should handicap one bidder at the expense of another.

In the same environment as Laffont and Robert, Gavious, Moldovanu and Sela (2000) compare the revenues to be realized from the standard auction forms. Che and Gale (1996) assume the valuations to be common knowledge but the budgets private information. In this environment they compare the revenues to be realized by the standard auction forms. In addition they examine policies like joint bidding and subsidies and conclude such policies can help to increase the revenue over the standard auction forms. Che and Gale (2000) assume a single buyer with private valuations and a private budget constraint and derive a revenue maximizing, Bayesian 
incentive compatible selling mechanism. Since the model involves a single bidder it sheds no light on the issue of competition amongst bidders.

In this paper we consider a problem with two bidders, independent private valuations, bidding for a divisible good. One buyer has no liquidity constraint (the unconstrained bidder), while the other has one. Furthermore, the identity and budget constraint of the constrained bidder is common knowledge. In this environment we derive two mechansims. One is revenue maximizing, dominant strategy incentive compatible and ex-post individually rational. The second is revenue maximizing, Bayesian incentive compatible and interim individually rational. In addition, we extend the analysis to the case when the budget is private information (but not the identity of the constrained biddder) but the constrained bidder must post a bond. In this case we show how this case reduces to the case when the budget is common knowledge.

All the mechanisms considered are subsidy free. However, as noted by Zheng (2001), offering subsidies may be profitable for the seller. In our model the effect of a subsidy can be computed by increasing the relevant budget constraint and determining if the increase in expected revenue is sufficient to cover the cost of the subsidy.

Two qualitative features of the derived mechanisms differ from the classic optimal mechanism of Myerson (1981) which assumes all bidders are unconstrained. Under a monotone hazard assumption on the distribution of types, Myerson shows that an optimal mechanism will allocate the good to the agent with the highest non-negative virtual value. In the environment considered here, the optimal mechanism may assign a portion of the good to the agent with the lowest virtual value. ${ }^{1}$ Furthermore, the good, in some cases, is allocated to an agent with a negative virtual value.

What drives the first difference is the following observation. Suppose the constrained bidder has a value/type $i$ and a budget $b$. As long as one can offer this bidder a quantity $q \geq b / i$ one can charge her $b$. Notice that no higher payment from the constrained bidder can be extracted by offering more than $b / i$. Hence, it may pay to allocate a portion to the unconstrained bidder even though she may have a lower virtual value. For the second

\footnotetext{
${ }^{1}$ Although we assume the good is divisible, one can interpret a fractional allocation as the probability of assiging an indivisible good. In this sense our results carry over to the case of a single indivisible good.
} 
difference, suppose the virtual value of both bidders is negative. It may still be profitable to offer the constrained bidder at least $q \geq b / i$ because one can extract $b$.

Perhaps the most important lesson to be learnt from the analysis is that the seller must sometimes withold the good from the unconstrained bidder even if she happens to be the highest bidder. In the event that the unconstrained bidder submits a bid that exceeds the budget of the constrained bidder, the mechanisms derived here, require that the high bid must exceed the budget by a fixed amount in order to secure the good.

Ther remainder of this paper is divided into three sections. One devoted to the dominant strategy incentive compatible case, the second to the Bayesian incentive compatible case and the third to the case of the budget being private information. In a departure from custom we assume a discrete type space. ${ }^{2}$ This allows us to employ simple arguments involving linear program's to derive the results. It is clear what the continuous analog's of our results are.

\section{The Dominant Strategy Incentive Compatible Case}

Let $\{1,2, \ldots, m\}$ be the set of possible types, $f_{i}>0$ the probability that a bidder is of type $i, F(i)=\sum_{j=1}^{i} f_{i}$ and $v_{i}=\left[i-\frac{1-F(i)}{f_{i}}\right]$ the virtual value of type $i$. We assume that virtual values are monotone in types (the hazard condition). We use $t$ to denote the type of the unconstrained bidder and $s$ the type of constrained bidder. Let $b$ be the known budget of the constrained bidder.

Our goal is to derive the dominant strategy incentive compatible, expost individually rational mechanism that maximizes expected revenue. As is standard, we invoke the revelation principle to restrict attention to direct revelation mechanisms. Let $a(t, s)$ be the allocation to the unconstrained bidder when the profile of reported types is $(t, s)$ and $a_{b}(t, s)$ the allocation to the constrained bidder when the profile of reported types is $(t, s)$. The payment that each makes at the profile $(t, s)$ is denoted by $P(t, s)$ and $P_{b}(t, s)$

\footnotetext{
${ }^{2}$ Nevertheless this is consistent with the early work on optimal mechanism design by Harris and Raviv (1981).
} 
respectively.

Dominant strategy incentive compatibility (IC) requires that

$$
\begin{gathered}
t a(t, s)-P(t, s) \geq t a\left(t^{\prime}, s\right)-P\left(t^{\prime}, s\right) \forall t^{\prime} \neq t, \\
s a_{b}(t, s)-P_{b}(t, s) \geq s a\left(t, s^{\prime}\right)-P_{b}\left(t, s^{\prime}\right) \forall s^{\prime} \neq s .
\end{gathered}
$$

Introducing a dummy type $i=0$ and invoking the subsidy free assumption allows us to set $P(0, s)=P_{b}(t, 0)=0$ and $a(0, s)=a_{b}(t, 0)=0$. This enables us to fold the individual rationality (IR) constraint into the (IC) constraints.

The next two results are standard so proofs are omitted.

Theorem 1 An allocation rule is dominant strategy incentive compatible iff it is monotonic. That is $a(t, s) \geq a\left(t^{\prime}, s\right)$ iff. $t \geq t^{\prime}$ and $a_{b}(t, s) \geq a_{b}\left(t, s^{\prime}\right)$ iff. $s \geq s^{\prime}$.

Theorem 2 All IC constraints are implied by the following:

$$
\begin{gathered}
t a(t, s)-P(t, s) \geq t a(t-1, s)-P(t-1, s) \forall t \leq m, \\
t a(t, s)-P(t, s) \geq t a(t+1, s)-P(t+1, s) \forall t \leq m-1 \\
s a_{b}(t, s)-P_{b}(t, s) \geq s a(t, s-1)-P_{b}(t, s-1) \forall s \leq m . \\
s a_{b}(t, s)-P_{b}(t, s) \geq s a_{b}(t, s+1)-P_{b}(t, s+1) \forall s \leq m-1
\end{gathered}
$$

The problem of finding the optimal auction is formulated as a linear 
program below.

$$
\begin{aligned}
& {[O P T] Z=\max _{P_{b}(t, s), P(t, s)} \sum_{t=0}^{m} \sum_{s=0}^{m} f_{t} f_{s}\left[P(t, s)+P_{b}(t, s)\right] } \\
& \text { s.t. } t a(t, s)-P(t, s) \geq t a(t-1, s)-P(t-1, s) \forall t \leq m \\
& \quad t a(t, s)-P(t, s) \geq t a(t+1, s)-P(t+1, s) \forall t \leq m-1 \\
& s a_{b}(t, s)-P_{b}(t, s) \geq s a(t, s-1)-P_{b}(t, s-1) \forall s \leq m \\
& s a_{b}(t, s)-P_{b}(t, s) \geq s a_{b}(t, s+1)-P_{b}(t, s+1) \forall s \leq m-1 \\
& a(t, s) \geq a(t-1, s) \forall t \leq m, s \\
& a_{b}(t, s) \geq a_{b}(t, s-1) \forall s \leq m, t \\
& P_{b}(t, s) \leq b \forall t, s \\
& a(t, s)+a_{b}(t, s) \leq 1 \forall t, s \\
& a(t, s), a_{b}(t, s) \geq 0 \forall t, s
\end{aligned}
$$

Fixing the $a$ 's, $a_{b}$ 's and the type $t$ of the unconstrained bidder, $P_{b}(t, \cdot)$ can be expressed as a shortest path length in an appropriate network.

In this network there is one vertex for each type $s$ of the constrained bidder, including the dummy type. The vertex corresponding to the dummy type is called the source vertex. For each type/vertex $i \geq 1$ there is a directed edge $(0, i)$ of length $b$ (only the edge from source to vertex $i$ is depicted). Between the source and vertex $\{1\}$ there are two parallel edges. One of length $a_{b}(t, 1)$ and the other of length $b$.

Between $i$ and $i+1$ there are two edges. One directed from $i$ to $i+1$ of length $(i+1)\left[a_{b}(t, i+1)-a_{b}(t, i)\right]$. Monotonicity of the allocation rule implies this edge has non-negative length. The other edge is directed from $i+1$ to $i$ and of length $i\left[a_{b}(t, i)-a_{b}(t, i+1)\right] \leq 0$. A portion of the network is depicted in Figure 1 (the edge of length $b$ from the source to vertex 1 is not depicted.) 


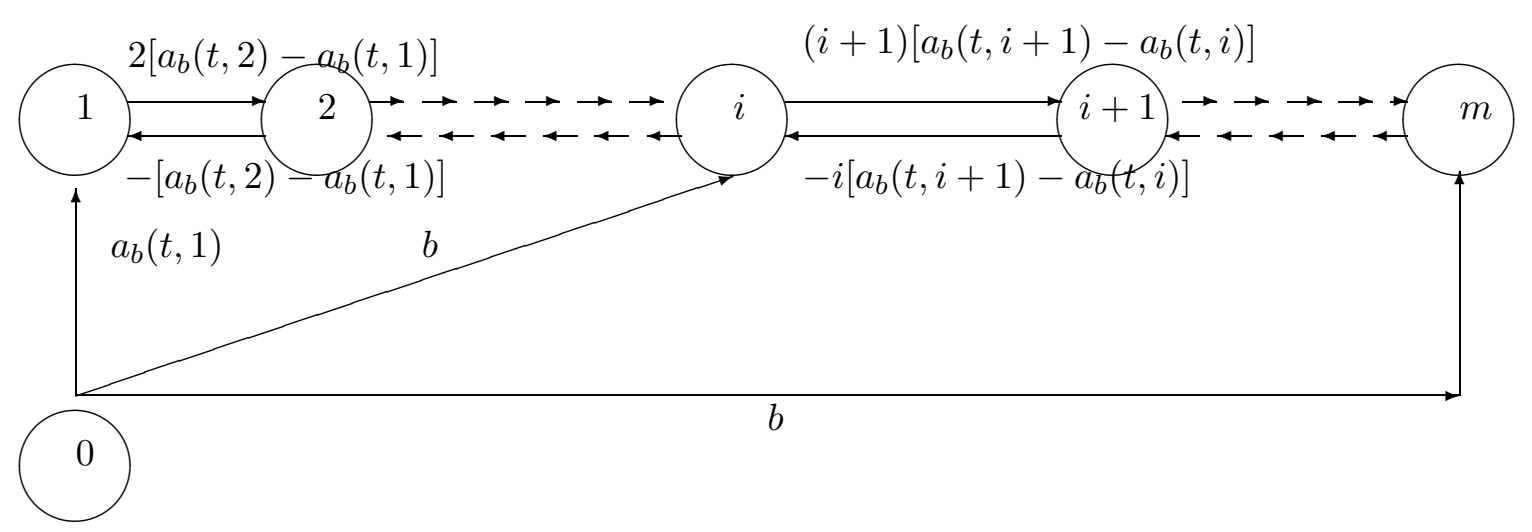

Figure 1

The edges directed from left to right correspond to 'downward' IC constraints while the edges in the opposte directions correspond to 'upward' IC constraints. An implication of monotonicty of the $a_{b}$ 's is that there are no negative cycles in the network.

The largest value that $P_{b}(t, i)$ can take is the length of the shortest path from the source to vertex $i$. The absence of negative length cycles means these shortest path lengths are well defined. In this network there are at most two candidates for the shortest path from the source to $i<m$. One from left to right and one from right to left. Let $L_{i}^{b}(t)$ be the length of the path from node 0 to node $i$ that is from left to right, and $R_{i}^{b}(t)$ be the length of the path from node 0 to node $i$ that is from right to left. In particular $L_{0}^{b}(t)=0, L_{i}^{b}(t)=\sum_{j=0}^{i-1}(j+1)\left[a_{b}(t, j+1)-a_{b}(t, j)\right] \forall i>0$. Similarly $R_{i}^{b}(t)=b-\sum_{j=i}^{m-1} j\left[a_{b}(t, j+1)-a_{b}(t, j)\right] \forall i<m$ and $R_{m}^{b}(t)=b$. Clearly, $P_{b}(t, i)=\min \left(L_{i}(t), R_{i}(t)\right)$.

A similar network can be constructed to determine $P(i, s)$. It will be identical to the one in Figure 1 except the edges of length $b$ from the source to each vertex $i$ will be absent. It is easy to see from an identical analysis 
that

$$
P(i, s)=\sum_{j=0}^{i-1}(j+1)[a(j+1, s)-a(j, s)] .
$$

We can rewrite $[O P T]$ as

$$
\begin{array}{ll} 
& Z=\max _{P_{b}(t, s), P(t, s)} \sum_{t=0}^{m} \sum_{s=0}^{m} f_{t} f_{s}\left[P(t, s)+P_{b}(t, s)\right] \\
\text { s.t. } & P_{b}(t, s)=\min \left\{L_{s}^{b}(t), R_{s}^{b}(t)\right\} \forall t, s \\
& P(t, s)=\sum_{j=0}^{t-1}(j+1)[a(j+1, s)-a(j, s)] \forall t, s \\
& a(t, s) \geq a(t-1, s) \forall t \leq m, s \\
& a_{b}(t, s) \geq a_{b}(t, s-1) \forall s \leq m, t \\
& a(t, s)+a_{b}(t, s) \leq 1 \forall t, s \\
& a(t, s), a_{b}(t, s) \geq 0 \forall t, s
\end{array}
$$

Straightforward algebra shows that

$$
\sum_{t=0}^{m} \sum_{s=0}^{m} f_{t} f_{s} P(t, s)=\sum_{t=0}^{m} \sum_{s=0}^{m} f_{t} f_{s}\left(\sum_{j=0}^{t-1}(j+1)[a(j+1, s)-a(j, s)]\right)=\sum_{s=0}^{m} f_{s}\left[\sum_{t=0}^{m} f_{t} v_{t} a(t, s)\right] .
$$

Substituting this into $[O P T]$ to:

$$
\begin{gathered}
Z=\max _{P_{b}(t, s), a(t, s)} \sum_{s=0}^{m} f_{s}\left[\sum_{t=0}^{m} f_{t} v_{t} a(t, s)\right]+\sum_{t=0}^{m} \sum_{s=0}^{m} f_{t} f_{s} P_{b}(t, s) \\
\text { s.t. } P_{b}(t, s)=\min \left\{L_{s}^{b}(t), R_{s}^{b}(t)\right\} \forall t, s \\
a(t, s) \geq a(t-1, s) \forall t \leq m, s \\
a_{b}(t, s) \geq a_{b}(t, s-1) \forall s \leq m, t \\
a(t, s)+a_{b}(t, s) \leq 1 \forall t, s \\
a(t, s), a_{b}(t, s) \geq 0 \forall t, s
\end{gathered}
$$

Monotonicity of the $a_{b}$ 's implies that $L_{i}^{b}(t) \leq L_{i+1}^{b}(t)$ and $b \geq R_{i}^{b}(t) \geq$ $R_{i+1}^{b}(t)$ for all $t$. Hence the budget constraint is already incorporated into the formulation. 
Consider now the following relaxation of $[O P T]$ which we call $[r O P T]$.

$$
\begin{gathered}
\max _{P_{b}(t, s), a(t, s)} \sum_{s=0}^{m} f_{s}\left[\sum_{t=0}^{m} f_{t} v_{t} a(t, s)\right]+\sum_{t=0}^{m} \sum_{s=0}^{m} f_{t} f_{s} P_{b}(t, s) \\
\text { s.t. } P_{b}(t, s) \leq L_{s}^{b}(t) \forall t, s \\
P_{b}(t, s) \leq b \forall t, s \\
a(t, s) \geq a(t-1, s) \forall t \leq m, s \\
a_{b}(t, s) \geq a_{b}(t, s-1) \forall s \leq m, t \\
a(t, s)+a_{b}(t, s) \leq 1 \forall t, s \\
a(t, s), a_{b}(t, s) \geq 0 \forall t, s
\end{gathered}
$$

Lemma 1 There is an optimal solution to $[r O P T]$ such that $L_{s}^{b}(t) \leq b$ for all $s$ and $t$.

\section{Proof}

Amongst all optimal solutiuons to $[r O P T]$ (this forms a compact set) pick one that minimizes $D=\sum_{t=1}^{m} \max \left\{L_{m}^{b}(t)-b, 0\right\}$. If $D=0$, monotonicity of the $a_{b}$ 's implies that $L_{s}^{b}(t) \leq L_{m}^{b}(t) \leq b$ for all $s$ and $t$ and the proof is complete. Suppose therefore that $D>0$. Hence there is a $t$ such that $L_{m}^{b}(t)>b$. Let $k$ be the smallest index such that $a_{b}(t, k)=a_{b}(t, k+1)=\ldots=a_{b}(t, m)$. Notice that $a_{b}(t, m)>0$. Construct a new solution by reducing $a_{b}(s, t)$ for all $s \geq k$ by $\epsilon>0$. Observe that this does not influence $P_{b}(t, s)$ for all $s$. For $s<k$ we have that $a_{b}(t, s)<a_{b}(t, k)$ and so for $\epsilon$ sufficiently small the new solution is still monotonic in the allocation variables. Thus, we have a new solution that is feasible, optimal but with a value of $D$ that is reduced, a contradiction.

In view of lemma 1 and the monotonicity of the $a$ 's we can write $[r O P T]$ 
as

$$
\begin{array}{r}
\max _{\left\{a(t, s), a_{b}(t, s)\right\}} \sum_{t} \sum_{s} f_{t} f_{s}\left[v_{t} a(t, s)+v_{s} a_{b}(t, s)\right] \\
\text { s.t. } a(t, s) \leq a(t+1, s) \forall t, s \\
a_{b}(t, s) \leq a_{b}(t, s+1) \forall t, s \\
a(t, s)+a_{b}(t, s) \leq 1 \forall t, s \\
a(t, s), a_{b}(t, s) \geq 0 \forall t, s \\
\sum_{s=0}^{m-1}(s+1)\left[a_{b}(t, s+1)-a_{b}(t, s)\right] \leq b \forall t
\end{array}
$$

Notice that any solution to this last program is feasible for $[O P T]$ as well. This is because $L_{s}^{b}(t) \leq b \Rightarrow L_{s}^{b}(t) \leq R_{s}^{b}(t)$.

\subsection{A Relaxation of problem (P)}

We relax problem $(\mathrm{P})$ by removing the monotonicity constraints on $a$.

$$
\begin{gathered}
\max _{\left\{a(t, s), a_{b}(t, s)\right\}} \sum_{t} \sum_{s} f_{t} f_{s}\left[v_{t} a(t, s)+v_{s} a_{b}(t, s)\right] \quad\left(P_{1}\right) \\
\text { s.t. } a_{b}(t, s) \leq a_{b}(t, s+1) \forall t, s \\
a(t, s)+a_{b}(t, s) \leq 1 \forall t, s \\
a(t, s), a_{b}(t, s) \geq 0 \forall t, s \\
\sum_{s=0}^{m-1}(s+1)\left[a_{b}(t, s+1)-a_{b}(t, s)\right] \leq b \forall t
\end{gathered}
$$

We will solve $\left(\mathrm{P}_{1}\right)$ and it will be verified that the solution satisfies the omitted monotonicity constraints. Thus the relaxation is exact.

Problem $\left(\mathrm{P}_{1}\right)$ itself can be decomposed into a collection of independent sub-problems indexed by the type $t$ of the unconstrained bidder:

$$
\begin{array}{r}
\max _{\left\{a(t, s), a_{b}(t, s)\right\}} \sum_{s} f_{s}\left[v_{t} a(t, s)+v_{s} a_{b}(t, s)\right] \\
\text { s.t. } a_{b}(t, s) \leq a_{b}(t, s+1) \forall s \\
a(t, s)+a_{b}(t, s) \leq 1 \forall s \\
m a_{b}(t, m)-\sum_{j=1}^{m-1} a_{b}(t, j) \leq b \forall t
\end{array}
$$


We divide the solution of $\left(\mathrm{P}_{t}\right)$ into two cases.

\subsection{Solution of $\left(\mathbf{P}_{t}\right)$ when $v_{t} \geq 0$}

We can deal with the monotonicity constraints on the $a_{b}$ 's by a change of variables. Let $a_{b}(t, s)=\sum_{i=1}^{s} \Delta(t, i)$ where $\Delta(t, i) \geq 0$ for all $i$. For convenience set $w_{s}=\sum_{j=s}^{m} f_{j} v_{j}$ for all $s$. Replacing the $a_{b}$ 's by $\Delta$ 's in problem $\left(\mathrm{P}_{t}\right)$ yields:

$$
\begin{array}{r}
\max _{\{a(t, s), \Delta(s, t)\}} \sum_{s=1}^{m} f_{s} v_{t} a(t, s)+\sum_{s=1}^{m} w_{s} \Delta(t, s) \\
\text { s.t. } a(t, s)+\sum_{i=1}^{s} \Delta(t, i) \leq 1 \forall s \\
\sum_{s=1}^{m} s \Delta(t, s) \leq b \\
a(t, s), \Delta(t, s) \geq 0 \forall s
\end{array}
$$

It is easy to see that there is an optimal solution to $\left(\mathrm{LP}_{t}\right)$ such that $a(t, s)+$ $\sum_{i=1}^{s} \Delta(t, i)=1$ for all $s$. If not, i.e. $a(t, s)+\sum_{i=1}^{s} \Delta(t, i)<1$ for some $s$, increase $a(t, s)$. Since $v_{t} \geq 0$ the objective function cannot decrease. Given this we can use the equation $a(t, s)+\sum_{i=1}^{s} \Delta(t, i)=1$ to substitute out the $a(t, s)$ variables. Specifically $a(t, s)=1-\sum_{i=1}^{s} \Delta(t, i)$ for all $s$. Therefore $\left(\mathrm{LP}_{t}\right)$ becomes:

$$
\begin{aligned}
& \max _{\{a(t, s), \Delta(s, t)\}} \sum_{s=1}^{m} f_{s} v_{t}\left[1-\sum_{i=1}^{s} \Delta(t, i)\right]+\sum_{s=1}^{m} w_{s} \Delta(t, s) \\
& \text { s.t. } \sum_{i=1}^{s} \Delta(t, i) \leq 1 \forall s \\
& \sum_{s=1}^{m} s \Delta(t, s) \leq b \\
& \Delta(t, s) \geq 0 \forall s
\end{aligned}
$$

A second observation is the constraints $\sum_{i=1}^{s} \Delta(t, i) \leq 1$ for all $s=1, \ldots, m-$ 1 are all implied by the constraint $\sum_{i=1}^{m} \Delta(t, i) \leq 1$. This allows us to reduce 
$\left(\mathrm{LP}_{t}\right)$ to

$$
\begin{array}{r}
\max _{\{a(t, s), \Delta(s, t)\}} \sum_{s=1}^{m} f_{s} v_{t}\left[1-\sum_{i=1}^{s} \Delta(t, i)\right]+\sum_{s=1}^{m} w_{s} \Delta(t, s) \\
\text { s.t. } \sum_{i=1}^{m} \Delta(t, i) \leq 1 \\
\sum_{s=1}^{m} s \Delta(t, s) \leq b \\
\Delta(t, s) \geq 0 \forall s
\end{array}
$$

Simplifying the objective function and introducing slack variables $u_{1}$ and $u_{2}$ to make all constraints hold at equality produces:

$$
\begin{gathered}
\max _{\{\Delta(t, s)\}} \sum_{s=1}^{m}\left[\sum_{i=s}^{m} f_{i}\left(v_{i}-v_{t}\right)\right] \Delta(t, s)+\sum_{s=1}^{m} f_{s} v_{t} \\
\text { s.t. } \sum_{s=1}^{m} \Delta(t, s)+u_{1}=1 \\
\sum_{s=1}^{m} s \Delta(t, s)+u_{2}=b \\
\Delta(t, s), u_{i} \geq 0 \forall s, i
\end{gathered}
$$

The extreme points of this last linear program fall into one of three categories because it has only two constraints.

1. Exactly one $i$ such that $\Delta(t, i)>0, u_{1}>0, u_{2}=0$.

2. Exactly one $i$ such that $\Delta(t, i)>0, u_{1}=0, u_{2}>0$.

3. Exactly one pair $(p, q)$ with $p \neq q, p \leq b \leq q, \Delta(t, p), \Delta(t, q)>0$ and $u_{1}=u_{2}=0$.

From each category we choose the extreme point with largest objective function value. The optimal solution must be the extreme point from the three chosen with largest objective function value. The best extreme point of each type is listed below. 


\section{Category 1:}

Let $r^{1}=\arg \max _{s \geq b} \frac{b \sum_{i=s}^{m} f_{i}\left(v_{i}-v_{t}\right)}{s}$.

Set $\Delta\left(t, r^{1}\right)=\frac{b}{r_{1}}, u_{1}=1-\frac{b}{r_{1}}$ and $u_{2}=0$.

The objective function value of this solution is $b \frac{\sum_{i=r^{1}}^{m} f_{i}\left(v_{i}-v_{t}\right)}{s}+\sum_{s=1}^{m} f_{s} v_{t}$.

\section{Category 2:}

Let $r^{2}=\arg \max _{s \leq b}\left[\sum_{i=s}^{m} f_{i}\left(v_{i}-v_{t}\right)\right]$. In fact, by the monotone hazard condition, when $b \leq t, r_{2}=b$ and when $b>t, r_{2}=t$.

Set $\Delta\left(t, r^{2}\right)=1, u_{1}=0$ and $u_{2}=b-r^{2}$.

The objective function value of this solution is $\sum_{i=r^{2}}^{m} f_{i}\left(v_{i}-v_{t}\right)+$ $\sum_{s=1}^{m} f_{s} v_{t}$.

\section{Category 3: Let}

$$
\left(p^{3}, q^{3}\right)=\arg \max _{p \leq b \leq q}\left[\frac{(q-b)}{(q-p)} \sum_{i=p}^{m} f_{i}\left(v_{i}-v_{t}\right)+\frac{(b-p)}{(q-p)} \sum_{i=q}^{m} f_{i}\left(v_{i}-v_{t}\right)\right] .
$$

Set $\Delta\left(t, p^{3}\right)=\frac{\left(q^{3}-b\right)}{\left(q^{3}-p^{3}\right)}, \Delta\left(t, q^{3}\right)=\frac{\left(b-p^{3}\right)}{\left(q^{3}-p^{3}\right)}$ and $u_{1}=u_{2}=0$.

In fact we can pin down the category 3 solution even further. Suppose first that $b \leq t$. Then, by the monotone hazard condition, $\sum_{i=q}^{m} f_{i}\left(v_{i}-v_{t}\right)$ is maximized when $q=t$ and $\sum_{i=p}^{m} f_{i}\left(v_{i}-v_{t}\right)$ is maximized when $p=b$. In this case the category 3 solution is $\Delta\left(t, p^{3}\right)=1$ and $\Delta\left(t, q^{3}\right)=0$, i.e. a category 2 solution. A similar argument applies in the case when $b>t$.

Therefore, only category 1 and 2 solutions apply.

Theorem 3 If $b>t$ then $\Delta(t, t)=1$ is optimal. If $b \leq t$ then $\Delta\left(t, r^{1}\right)=\frac{b}{r_{1}}$ is optimal.

\section{Proof}

Suppose first $b>t$. By the monotone hazard condition, $\frac{\sum_{i=s}^{m} f_{i}\left(v_{i}-v_{t}\right)}{s}$ is non-increasing for $s \geq t$. Hence

$$
\arg \max _{s \geq b} \frac{b \sum_{i=s}^{m} f_{i}\left(v_{i}-v_{t}\right)}{s}=b .
$$

In this case the objective function value of the category 1 solution is $\sum_{s=b}^{m} f_{s}\left(v_{s}-\right.$ $\left.v_{t}\right)$. However the category 2 solution has objective function value $\sum_{s=t}^{m} f_{s}\left(v_{s}-\right.$ $v_{t}$ ), which is larger. 
Now suppose $b \leq t$. The catgeory 2 solution has objective function value $\sum_{s=b}^{m} f_{s}\left(v_{s}-v_{t}\right)$. But this is bounded above by $\max _{s \geq b} \frac{b \sum_{i=s}^{m} f_{i}\left(v_{i}-v_{t}\right)}{s}$, i.e., the category 1 solution is optimal.

To summarize:

1. If $b>t$ then $a_{b}(t, s)=1, a(t, s)=0$ for all $s \geq t$ and $a_{b}(t, s)=$ $0, a(t, s)=1$ otherwise.

2. If $b \leq t$ then $a_{b}(t, s)=\frac{b}{r^{1}}, a(t, s)=1-\frac{b}{r^{1}}$ for all $s \geq r^{1}$ and $a_{b}(t, s)=$ $0, a(t, s)=1$ otherwise.

Using the expressions for for the payment variables in terms of path lengths we can compute the payments bidders must make. The allocations are summarized in the table below.

\begin{tabular}{|c|c|c|c|}
\hline$v_{t} \geq 0$ & $s \geq r^{1}$ & $b \leq s \leq r^{1}$ & $s \leq b-1$ \\
\hline$t \geq b$ & $a(t, s)=\left(1-\frac{b}{r^{1}}\right)$ & $a(t, s)=1$ & $a(t, s)=1$ \\
& $a_{b}(t, s)=\frac{b}{r^{1}}$ & $a_{b}(t, s)=0$, & $a_{b}(t, s)=0$ \\
\hline$t \leq b-1$ & $a(t, s)=0$ & $a(t, s)=0$, & usual auction rules \\
& $a_{b}(t, s)=1$ & $a_{b}(t, s)=1$ & \\
\hline
\end{tabular}

Examination of the table shows that $a$ satisfies the omitted montonicity constraint.

\subsection{Solution of $\left(\mathbf{P}_{t}\right)$ when $v_{t}<0$}

In problem $\left(\mathrm{P}_{t}\right)$ if $v_{t}<0$ then $a(t, i)=0$ for all $i$ in any optimal solution. Note that if $v_{i}<0$ for some $i$ we cannot conclude that $a_{b}(t, i)=0$ in every optimal solution because of the budget constraint. Problem $\left(\mathrm{P}_{t}\right)$ becomes

$$
\begin{aligned}
& \max _{\{\Delta(s, t)\}} \sum_{i=1}^{m} w_{s}^{t} \Delta(t, s) \\
& \text { s.t. } \sum_{i=1}^{s} \Delta(t, i) \leq 1 \forall s \\
& \sum_{s=1}^{m} s \Delta(t, s) \leq b \\
& \Delta(t, s) \geq 0 \forall s
\end{aligned}
$$


The similarity of $\left(\mathrm{LP}_{t}\right)$ to $\left(\mathrm{LP}_{t}\right)$ permits an identical analysis whose details are omitted. The conclusions are summarized below. Let $h^{1}=\arg \max _{s \geq b} \frac{b \sum_{i=s}^{m} w_{i}}{s}$.

1. If $w_{1} \geq b \frac{w_{h^{1}}}{h^{1}}$ then $a_{b}(t, s)=1, a(t, s)=0$ for all $s \geq 1$ and $a_{b}(t, s)=$ $0, a(t, s)=0$ otherwise.

2. If $w_{1}<b \frac{w_{h^{1}}}{h^{1}}$ then $a_{b}(t, s)=\frac{b}{h^{1}}, a(t, s)=0$ for all $s \geq h^{1}$ and $a_{b}(t, s)=$ $0, a(t, s)=0$ otherwise.

Notice that the $a$ satisfy the omitted monotonicity constraint.

\section{The Bayesian Incentive Compatible Case}

Here we derive the revenue maximizing auction that is Bayesian incentive compatible and interim individually rational. Let $\mathcal{A}_{i}^{b}$ be the expected quantity of the good that the constrained agent receives when she reports type $i$ and all other agents report truthfully. Let $p_{i}^{b}$ be the expected payment of the constrained agent who reports type $i$. Similarly, $\mathcal{A}_{i}$ and $p_{i}$ are the expected allocations and payments for the unconstrained agent who reports type $i$. Notice that $\mathcal{A}_{i}^{b}=\sum_{t} f_{t} a_{b}(t, i)$ and $p_{i}^{b}=\sum_{t=1}^{m} f_{t} P_{b}(t, i)$ for all $i$. Similar expressions hold for $\mathcal{A}_{i}$ and $p_{i}^{u}$. Bayesian incentive compatibility (BIC) for the constrained agent requires:

$$
i \mathcal{A}_{i}^{b}-p_{i}^{b} \geq i \mathcal{A}_{j}^{b}-p_{j}^{b} \forall j \neq i .
$$

A similar inequality holds for the unconstrained agent.

Introducing a dummy type $i=0$ with $p_{0}^{u}, p_{0}^{u}=0$ and $\mathcal{A}_{0}^{b}, \mathcal{A}_{0}^{u}=0$ allows us to fold the interim individual rationality constraint into the (BIC) constraints.

From now on statements about $\mathcal{A}_{i}$ and $p_{i}$ are to be read as applying to $\mathcal{A}_{i}^{b}$ and $p_{i}^{b}$ as well.

The next two results are standard.

Theorem 4 An allocation rule that is incentive compatible iff it is monotonic. That is $r \leq s$ iff $\mathcal{A}_{r} \leq \mathcal{A}_{s}$ for all $k=1, \ldots, m$.

Theorem 5 All (BIC) constraints are implied by the following:

$$
\begin{gathered}
i \mathcal{A}_{i}-p_{i} \geq i \mathcal{A}_{i-1}-p_{i-1} \forall i=1, \ldots, m \\
i \mathcal{A}_{i}-p_{i} \geq i \mathcal{A}_{i+1}-p_{i+1} \forall i=1, \ldots, m-1
\end{gathered}
$$


We can formulate the problem of finding the optimal auction as:

$$
[B O P T] Z=\max _{p_{i}, p_{i}^{b}} \sum_{s, t}^{m} f_{t} f_{s}\left(p_{t}+p_{s}^{b}\right)
$$

s.t. $i \mathcal{A}_{i}-p_{i} \geq i \mathcal{A}_{i-1}-p_{i-1} \forall i=1, \ldots, m$

$$
\begin{gathered}
i \mathcal{A}_{i}-p_{i} \geq i \mathcal{A}_{i+1}-p_{i+1} \forall i=1, \ldots, m-1 \\
\mathcal{A}_{i}=\sum_{s} f_{s} a(i, s) \forall i \\
\mathcal{A}_{i}^{b}=\sum_{t} f_{t} a_{b}(t, i) \forall i \\
p_{i}=\sum_{s=1}^{m} f_{t} P(i, s) \forall i \\
p_{i}^{b}=\sum_{t=1}^{m} f_{t} P_{b}(t, i) \forall i \\
a(t, s)+a_{b}(t, s) \leq 1 \forall t, s \\
p_{i}^{b} \leq b \forall i
\end{gathered}
$$

The last constraint requires that the expected payment not exceed $b$.

As in the previous case there is a representation of the BIC constraints that allows one to interpret the $P_{i}$ 's as shortest path lengths in a network. Specifically,

$$
p_{s}^{b}=\min \left\{\sum_{j=0}^{s-1}(j+1)\left(\mathcal{A}_{j+1}^{b}-\mathcal{A}_{j}^{b}\right), b-\sum_{j=i}^{m-1} j\left[\mathcal{A}_{j+1}^{b}-\mathcal{A}_{j}^{b}\right]\right\}
$$

and

$$
p_{t}=\sum_{j=0}^{t-1}(j+1)\left(\mathcal{A}_{j+1}-\mathcal{A}_{j}\right)
$$


for all $s, t$. An analysis identical to the dominant strategy case allows us to write $[B O P T]$ as

$$
\begin{array}{r}
\max \sum_{s, t}\left[f_{t}\left(\sum_{j=0}^{t-1}(j+1)\left(\mathcal{A}_{j+1}-\mathcal{A}_{j}\right)\right)+f_{s}\left(\sum_{j=0}^{s-1}(j+1)\left(\mathcal{A}_{j+1}^{b}-\mathcal{A}_{j}^{b}\right)\right)\right] \\
\text { s.t. } \sum_{j=0}^{i-1}(j+1)\left(\mathcal{A}_{j+1}^{b}-\mathcal{A}_{j}^{b}\right) \leq b \forall i>0 \\
\mathcal{A}_{1} \leq \ldots \leq \mathcal{A}_{m} \\
\mathcal{A}_{i}=\sum_{s} f_{s} a(i, s) \forall i \\
\mathcal{A}_{i}^{b}=\sum_{t} f_{t} a_{b}(t, i) \forall i \\
a(t, s)+a_{b}(t, s) \leq 1 \forall t, s \\
a(t, s), a_{b}(t, s) \geq 0 \forall t, s
\end{array}
$$

Collecting like terms in the objective function together shows that

$\sum_{s, t}\left[f_{t}\left(\sum_{j=0}^{t-1}(j+1)\left(\mathcal{A}_{j+1}-\mathcal{A}_{j}\right)\right)+f_{s}\left(\sum_{j=0}^{s-1}(j+1)\left(\mathcal{A}_{j+1}^{b}-\mathcal{A}_{j}^{b}\right)\right)\right]=\sum_{s, t}\left[f_{t} \mathcal{A}_{t} v_{t}+f_{s} \mathcal{A}_{s}^{b} v_{s}\right]$.

The constraints $\sum_{j=0}^{i-1}(j+1)\left(\mathcal{A}_{j+1}^{b}-\mathcal{A}_{j}^{b}\right) \leq b$ for all $i>0$ can be rewritten to read $i \mathcal{A}_{i} \leq b+\sum_{j=1}^{i-1} \mathcal{A}_{j}$. For each pair $i$ label the constraint $i \mathcal{A}_{i}^{b} \leq$ $b+\sum_{j=1}^{i-1} \mathcal{A}_{j}^{b}$ as $C_{i}$.

Problem $[B O P T]$ becomes:

$$
\begin{array}{r}
Z=\max \sum_{s, t}\left[f_{t} \mathcal{A}_{t} v_{t}+f_{s} \mathcal{A}_{s}^{b} v_{s}\right] \\
\text { s.t. } i \mathcal{A}_{i}^{b} \leq b+\sum_{j=1}^{i-1} \mathcal{A}_{j}^{b} \forall i \\
0 \leq \mathcal{A}_{1} \leq \ldots \leq \mathcal{A}_{m} \\
\mathcal{A}_{i}=\sum_{s} f_{s} a(i, s) \forall i \\
\mathcal{A}_{i}^{b}=\sum_{t} f_{t} a_{b}(t, i) \forall i \\
a(t, s)+a_{b}(t, s) \leq 1 \forall t, s \\
a(t, s), a_{b}(t, s) \geq 0 \forall t, s
\end{array}
$$


Lemma 2 Suppose in some optimal solution to $[B O P T]$ one or more of the $C_{i}$ are binding. Let $s^{*}$ be the smallest index for which $C_{i}$ is binding. Then there is an optimal solution to $[B O P T]$ such that

$$
\mathcal{A}_{m}^{b}=\ldots=\mathcal{A}_{s^{*}}^{b}
$$

\section{Proof}

Since $C_{s^{*}}$ is binding we have $s^{*} \mathcal{A}_{s^{*}}^{b}=b+\sum_{j=1}^{s^{8}-1} \mathcal{A}_{j}^{b}$. From $C_{s^{*}+1}$ we have

$$
\left(s^{*}+1\right) \mathcal{A}_{s^{*}+1}^{b} \leq b+\sum_{j=1}^{s^{*}} \mathcal{A}_{j}^{b}=\mathcal{A}_{s^{*}}^{b}+s^{*} \mathcal{A}_{s^{*}}^{b}=\left(s^{*}+1\right) \mathcal{A}_{s^{*}}^{b} .
$$

But $\mathcal{A}_{s^{*}+1}^{b} \geq \mathcal{A}_{s^{*}}^{b}$, which implies that $\mathcal{A}_{s^{*}+1}^{b}=\mathcal{A}_{s^{*}}^{b}$. Thus $C_{s^{*}+1}$ is binding and we repeat the argument for index $s^{*}+2$ and so on.

Suppose we knew the critical index $s^{*}$ from the lemma. Consider the following optimization problem $\left[B O P T\left(s^{*}\right)\right]$ :

$$
\begin{array}{r}
Z\left(s^{*}\right)=\max \sum_{s, t}\left[f_{t} \mathcal{A}_{t} v_{t}+f_{s} \mathcal{A}_{s}^{b} v_{s}\right] \\
\text { s.t. } \mathcal{A}_{1}^{b} \leq \ldots \leq \mathcal{A}_{s^{*}}^{b}=\ldots=\mathcal{A}_{m}^{b} \\
\mathcal{A}_{1} \leq \ldots \leq \mathcal{A}_{m} \\
\mathcal{A}_{i}=\sum_{s} f_{s} a(i, s) \forall i \\
\mathcal{A}_{i}^{b}=\sum_{t} f_{t} a_{b}(t, i) \forall i \\
a(t, s)+a_{b}(t, s) \leq 1 \forall t, s \\
a(t, s), a_{b}(t, s) \geq 0 \forall s, t
\end{array}
$$

It is clear that $Z=Z\left(s^{*}\right)$. We will show that there is an optimal solution to $\left[B O P T\left(s^{*}\right)\right]$ such that $a_{b}(t, s)=a_{b}\left(t, s^{*}\right)$ for all $t$ and $s \geq s^{*}$. We do this 
by examining a relaxation of problem $\left[B O P T\left(s^{*}\right)\right]$ called $\left[R O P T\left(s^{*}\right)\right]$.

$$
\begin{array}{r}
Z^{r}\left(s^{*}\right)=\max \sum_{s, t}\left[f_{t} \mathcal{A}_{t} v_{t}+f_{s} \mathcal{A}_{s}^{b} v_{s}\right] \\
\text { s.t. } \mathcal{A}_{s^{*}}^{b}=\ldots=\mathcal{A}_{m}^{b} \\
\mathcal{A}_{i}=\sum_{s} f_{s} a(i, s) \forall i \\
\mathcal{A}_{i}^{b}=\sum_{t} f_{t} a_{b}(t, i) \forall i \\
a(t, s)+a_{b}(t, s) \leq 1 \forall t, s \\
a(t, s), a_{b}(t, s) \geq 0 \forall s, t
\end{array}
$$

The relaxtion is obtained by removing the monontonicity constraints on $\mathcal{A}_{j}$ for all $j$, the monotonicity constraints on $\mathcal{A}_{j}^{b}$ for $j=1, \ldots, s^{*}-1$. We show that there is an optimal solution to the relaxation that satisfies the omitted constraints, so making the relxation exact.

\subsection{Solving $\left[R O P T\left(s^{*}\right)\right]$}

The Lagrangean dual of $\left[R O P T\left(s^{*}\right)\right]$ is problem $\left(\mathrm{P}_{\lambda}\right)$, shown below.

$$
\begin{array}{r}
Z_{\lambda}\left(s^{*}\right)=\max \sum_{s, t}\left[f_{t} \mathcal{A}_{t} v_{t}+f_{s} \mathcal{A}_{s}^{b} v_{s}\right]+\sum_{j=s^{*}}^{m-1} \lambda_{j}\left(\mathcal{A}_{j+1}^{b}-\mathcal{A}_{j}^{b}\right) \\
\text { s.t. } \mathcal{A}_{i}=\sum_{s} f_{s} a(i, s) \forall i \\
\mathcal{A}_{i}^{b}=\sum_{t} f_{t} a_{b}(t, i) \forall i \\
a(t, s)+a_{b}(t, s) \leq 1 \forall t, s \\
a(t, s), a_{b}(t, s) \geq 0 \forall s, t
\end{array}
$$

By the duality theorem, $Z^{r}\left(s^{*}\right)=\min _{\lambda} Z_{\lambda}\left(s^{*}\right)$.

The objective function of $\left(\mathrm{P}_{\lambda}\right)$ can be written as

$$
\sum_{t=1}^{m} f_{t} \mathcal{A}_{t} v_{t}+\sum_{s=1}^{s^{*}-1} f_{s} \mathcal{A}_{s}^{b} v_{s}+\sum_{s=s^{*}}^{m} f_{s}\left[v_{s}+\frac{\lambda_{s-1}}{f_{s}}-\frac{\lambda_{s}}{f_{s}}\right] \mathcal{A}_{s}^{b}
$$


where $\lambda_{s^{*}-1}=\lambda_{m}=0$. Substituting $\mathcal{A}_{i}=\sum_{s} f_{s} a(i, s)$ and $\mathcal{A}_{i}^{b}=\sum_{t} f_{t} a_{b}(t, i)$ into this expression yields:

$\sum_{t=1}^{m} \sum_{s=1}^{m} f_{t} v_{t} f_{s} a(t, s)+\sum_{s=1}^{s^{*}-1} \sum_{t=1}^{m} f_{s} v_{s} f_{t} a_{b}(t, s)+\sum_{s=s^{*}}^{m} \sum_{t=1}^{m} f_{s}\left[v_{s}+\frac{\lambda_{s-1}}{f_{s}}-\frac{\lambda_{s}}{f_{s}}\right] f_{t} a_{b}(t, s)$.

To simplify, let $h_{s}(\lambda)=v_{s}+\frac{\lambda_{s-1}}{f_{s}}-\frac{\lambda_{s}}{f_{s}}$ for $s \geq s^{*}$. Therefore

$$
\begin{array}{r}
Z_{\lambda}\left(s^{*}\right)=\max \sum_{t=1}^{m} \sum_{s=1}^{m} f_{t} v_{t} f_{s} a(t, s)+\sum_{s=1}^{s^{*}-1} \sum_{t=1}^{m} f_{s} v_{s} f_{t} a_{b}(t, s)+\sum_{s=s^{*}}^{m} \sum_{t=1}^{m} f_{s} h_{s}(\lambda) f_{t} a_{b}(t, s) \\
\text { s.t. } a(t, s)+a_{b}(t, s) \leq 1 \forall t, s \\
a(t, s), a_{b}(t, s) \geq 0 \forall s, t
\end{array}
$$

This decomposes into a collection of subproblems, one subproblem for each profile $(t, s)$ of types. When $s \leq s^{*}-1$ the subproblem is

$$
\begin{gathered}
g_{\lambda}(t, s)=\max v_{t} a(t, s)+v_{s} a_{b}(t, s) \\
\text { s.t. } a(t, s)+a_{b}(t, s) \leq 1 \\
a(t, s), a_{b}(t, s) \geq 0
\end{gathered}
$$

and when $s \geq s^{*}$ it is

$$
\begin{gathered}
g_{\lambda}(t, s)=\max v_{t} a(t, s)+h_{s}(\lambda) a_{b}(t, s) \\
\text { s.t. } a(t, s)+a_{b}(t, s) \leq 1 \\
a(t, s), a_{b}(t, s) \geq 0
\end{gathered}
$$

It is easy to see that for $s \leq s^{*}-1$ that $g_{\lambda}(t, s)=\max \left\{v_{t}, v_{s}\right\}$ and when $s \geq s^{*}, g_{\lambda}(t, s)=\max \left\{v_{t}, h_{s}(\lambda)\right\}$.

Therefore $Z_{\lambda}\left(s^{*}\right)=\sum_{t, s} f_{t} f_{s} g_{\lambda}(t, s)$. This allows us to formulate $\min _{\lambda} Z_{\lambda}\left(s^{*}\right)$ as a linear program $\left(\mathrm{LP}_{\lambda}\right)$ :

$$
\begin{array}{r}
\min \sum_{t, s} f_{t} f_{s} W(t, s) \\
\text { s.t. } W(t, s) \geq v_{t} \forall t \\
W(t, s) \geq v_{s} \forall s \leq s^{*}-1 \\
W(t, s) \geq h_{s}(\lambda) \forall s \geq s^{*} \\
\lambda_{s^{*}-1}, \lambda_{m}=0
\end{array}
$$


Lemma 3 There is an optimal solution $\left(W^{*}, \lambda^{*}\right)$ of $\left(L P_{\lambda}\right)$ such that $v_{s^{*}-1} \leq$ $h_{s^{*}}^{*} \leq \ldots \leq h_{m}^{*}$ where $h_{s}^{*}=v_{s}+\frac{\lambda_{s-1}^{*}}{f_{s}}-\frac{\lambda_{s}^{*}}{f_{s}}$ for $s \geq s^{*}$.

\section{Proof}

Denote the discrepancy of an optimal solution $\lambda^{*}$ to $\left(\operatorname{LP}_{\lambda}\right)$ by $\max \left\{v_{s^{*}-1}-\right.$ $\left.h_{s^{*}}^{*}, 0\right\}+\sum_{i \geq s^{*}+1} \max \left\{h_{i-1}^{*}-h_{i}^{*}, 0\right\}$. Amongst all optimal solutions to $\left(\operatorname{LP}_{\lambda}\right)$ pick the one that has the smallest discrepancy. If the discrepancy is zero, we are done. So, suppose not.

Case 1: There exits at least one $j \geq s^{*}+1$, such that $h_{j-1}^{*}>h_{j}^{*}$. If there exist more than one $j$ such that $h_{j-1}^{*}>h_{j}^{*}$, choose the largest $j$, for which $h_{j-1}^{*}>h_{j}^{*}$.

Let $l$ be the largest index such that $h_{j}^{*}=h_{j+1}^{*}=\ldots=h_{l}^{*}<h^{*} l+1{ }^{3}$ Suppose $l<m$.

We construct a contradiction by considering a new set of $\left\{\lambda_{i}^{\prime}\right\}_{i=0}^{m}$, such that

$$
\begin{aligned}
\lambda_{j-1}^{\prime} & =\lambda_{j-1}^{*}+\varepsilon, \\
\lambda_{i}^{\prime} & =\lambda_{i}^{*}-\varepsilon \quad \forall i \in[j, l], \\
\lambda_{i}^{\prime} & =\lambda_{i}^{*} \quad \forall i \in[1, j-2] \cup[l+1, m] .
\end{aligned}
$$

Denote $h_{i}^{\prime}=i-\frac{1-F(i)}{f_{i}}-\frac{\lambda_{i}^{\prime}}{f_{i}}+\frac{\lambda_{i-1}^{\prime}}{f_{i}}$ for all $i$. This change results in the following changes to the values of $\left\{h_{i}^{*}\right\}_{i=0}^{m}$ :

$$
\begin{aligned}
h_{j-1}^{\prime} & =h_{j-1}^{*}-\frac{\varepsilon}{f_{j-1}}, \\
h_{j}^{\prime} & =h_{j}^{*}+2 \frac{\varepsilon}{f_{j}}, \\
h_{l+1}^{\prime} & =h_{l+1}^{*}-\frac{\varepsilon}{f_{l+1}}, \\
h_{i}^{\prime} & =h_{i}^{*} \quad \forall i \notin\{j-1, j, l+1\} .
\end{aligned}
$$

Denote the change in the $\left(\mathrm{LP}_{\lambda}\right)$ problem objective function from changing $\lambda^{*}$ to $\lambda^{\prime}$ by $\Delta Z_{\lambda}\left(s^{*}\right)$. Consider the pairs $(t, s)$, for which $W(t, s)$ are affected by this change.

For $\varepsilon>0$ sufficiently small, decreasing $h_{j-1}^{*}$ by $\frac{\varepsilon}{f_{j-1}}$ affects $W(s, t)$ only if $v_{t}<h_{j-1}^{*}$. Similarly the decrease of $h_{l+1}^{*}$ by $\frac{\varepsilon}{f_{l+1}}$ affects $W(t, s)$ if $v_{t}<h_{l+1}^{*}$.

\footnotetext{
${ }^{3}$ It is possible that $l=j$.
} 
For $\varepsilon>0$ sufficiently small, increasing $h_{j}^{*}$ by $2 \frac{\varepsilon}{f_{j}}$ affects $W(t, s)$ only if $v_{t} \leq h_{j}$.

Therefore the change in the objective function $\Delta Z_{\lambda}\left(s^{*}\right)$ is:

$\Delta Z_{\lambda}\left(s^{*}\right) \leq 2 \varepsilon\left[\sum\left\{f_{t}: v_{t} \leq h_{j}^{*}\right\}\right]-\varepsilon\left[\sum\left\{f_{t}: v_{t}<h_{j-1}^{*}\right\}\right]-\varepsilon \sum\left\{f_{t}: v_{t}<\left(h_{l+1}^{*}\right\}\right]$.

Hence $\Delta Z_{\lambda}\left(s^{*}\right) \leq 0$, and we conclude that $\lambda^{\prime}$ is also an optimal solution to $\left(\mathrm{LP}_{\lambda}\right)$.

Computing the change in discrepancy from $\lambda^{*}$ to $\lambda^{\prime}$ we observe that $\max \left\{h_{j-2}-h_{j-1}, 0\right\}$ can increase by at most $\varepsilon / f_{j-1}$, the term $\max \left\{h_{j-1}-\right.$ $\left.h_{j}, 0\right\}$ goes down by $\varepsilon / f_{j-1}+2 \varepsilon / f_{j}$, the term $\max \left\{h_{j}-h_{j+1}, 0\right\}$ goes up by $2 \varepsilon / f_{j}$, and the term $\max \left\{h_{l}-h_{l+1}, 0\right\}$ goes down by $\varepsilon / f_{l+1}$. The contribution to discrepancy from other terms is unchanged. ${ }^{4}$ Notice that the discrepancy changes by $\varepsilon / f_{j-1}-\left(\varepsilon / f_{j-1}+2 \varepsilon / f_{j}\right)+2 \varepsilon / f_{j}-\varepsilon / f_{l+1}<0$, contradicting our choice of $\lambda^{*}$ as the one with the smallest discrepancy.

Now suppose that $l=m$. This implies that $h_{j}^{*}=h_{j+1}^{*}=\ldots=h_{m}^{*}{ }^{5}$

We construct a contradiction by considering a new set of $\left\{\lambda_{i}^{\prime}\right\}_{i=0}^{m}$, such that

$$
\begin{aligned}
& \lambda_{i}^{\prime}=\lambda_{i}^{*}+\varepsilon \quad \forall i \in[j-1, m-1], \\
& \lambda_{i}^{\prime}=\lambda_{i}^{*} \quad \forall i \notin[j-1, m-1] .
\end{aligned}
$$

Denote $h_{i}^{\prime}=i-\frac{1-F(i)}{f_{i}}-\frac{\lambda_{i}^{\prime}}{f_{i}}+\frac{\lambda_{i-1}^{\prime}}{f_{i}}$ for all $i$. This change results in the following changes to the values of $\left\{h_{i}^{*}\right\}_{i=0}^{m}$ :

$$
\begin{aligned}
h_{j-1}^{\prime} & =h_{j-1}^{*}-\frac{\varepsilon}{f_{j-1}}, \\
h_{m}^{\prime} & =h_{m}^{*}+\frac{\varepsilon}{f_{m}}, \\
h_{i}^{\prime} & =h_{i}^{*} \quad \forall i \notin\{j-1, m\} .
\end{aligned}
$$

For $\varepsilon>0$ sufficiently small, decreasing $h_{j-1}^{*}$ by $\frac{\varepsilon}{f_{j-1}}$ affects $W(t, s)$ only if $v_{t}<h_{j-1}^{*}$. Increasing $h_{m}^{*}$ by $\frac{\varepsilon}{f_{m}}$ affects $W(t, s)$ only if $v_{t} \leq h_{m}^{*}$.

The change in the objective function $\Delta Z_{\lambda}\left(s^{*}\right)$ is:

$$
\left.\Delta Z_{\lambda}\left(s^{*}\right) \leq \varepsilon\left[\sum\left\{f_{t}: v_{t} \leq h_{m}^{*}\right\}\right]-\varepsilon \sum\left\{f_{t}: v_{t}<h_{j-1}^{*}\right\}\right] \leq 0 .
$$

\footnotetext{
${ }^{4}$ the term $\max \left\{h_{j}-h_{j+1}, 0\right\}$ is also unchanged for a small enough $\varepsilon>0$.

${ }^{5}$ It is possible that $j=m$.
} 
This contradicts the optimality of $\lambda^{*}$ if $\Delta Z<0$, or implies that $\lambda^{\prime}$ is also an optimal solution to $\left(\mathrm{LP}_{\lambda}\right)$ if $\Delta Z_{\lambda}\left(s^{*}\right)=0$. In the latter case, we compute the change in discrepancy from $\lambda^{*}$ to $\lambda^{\prime}$. Notice that $\max \left\{h_{j-2}-h_{j-1}, 0\right\}$ (or $\max \left\{v_{s^{*}-1}-h_{s^{*}}, 0\right\}$ if $j=s^{*}+1$ ) can increase by at most $\varepsilon / f_{j-1}$, and the term $\max \left\{h_{j-1}-h_{j}, 0\right\}$ goes down by $\varepsilon / f_{j-1}+\varepsilon / f_{j}$. The contribution to discrepancy from other terms is unchanged. Hence the discrepancy changes by $\varepsilon / f_{j-1}-\left(\varepsilon / f_{j-1}+\varepsilon / f_{j}\right)=-\varepsilon / f_{j}<0$, contradicting our choice of $\lambda^{*}$ as the optimal solution with the smallest discrepancy.

Case 2: $h_{s^{*}}^{*} \leq h_{s^{*}+1}^{*} \leq \ldots \leq h_{m}^{*}$ but $v_{s^{*}-1}>h_{s^{*}}^{*}$. Let $l$ be the largest index such that $h_{s^{*}}^{*}=h_{s^{*}+1}^{*}=\ldots=h_{l}^{*}<h^{*} l+1$.

We construct a contradiction by considering a new set of $\left\{\lambda_{i}^{\prime}\right\}_{i=0}^{m}$, such that

$$
\begin{aligned}
& \lambda_{i}^{\prime}=\lambda_{i}^{*}-\varepsilon \quad \forall i \in\left[s^{*}, l\right], \\
& \lambda_{i}^{\prime}=\lambda_{i}^{*} \quad \forall i \in[1, j-2] \cup[l+1, m] .
\end{aligned}
$$

Denote $h_{i}^{\prime}=i-\frac{1-F(i)}{f_{i}}-\frac{\lambda_{i}^{\prime}}{f_{i}}+\frac{\lambda_{i-1}^{\prime}}{f_{i}}$ for all $i$. This change results in the following changes to the values of $\left\{h_{i}^{*}\right\}_{i=0}^{m}$ :

$$
\begin{aligned}
h_{s^{*}}^{\prime} & =h_{s^{*}}^{*}+\frac{\varepsilon}{f_{j}}, \\
h_{l+1}^{\prime} & =h_{l+1}^{*}-\frac{\varepsilon}{f_{l+1}}, \\
h_{i}^{\prime} & =h_{i}^{*} \quad \forall i \notin\left\{s^{*}, l+1\right\} .
\end{aligned}
$$

Denote the change in the $\left(\mathrm{LP}_{\lambda}\right)$ problem objective function from changing $\lambda^{*}$ to $\lambda^{\prime}$ by $\Delta Z_{\lambda}\left(s^{*}\right)$. Consider the pairs $(t, s)$, for which $W(t, s)$ are affected by this change.

For $\varepsilon>0$ sufficiently small, increasing $h_{s^{*}}^{*}$ by $\frac{\varepsilon}{f_{s^{*}}}$ affects $W(t, s)$ only if $v_{t} \leq h_{s^{*}}$. Similarly, the decrease of $h_{l+1}^{*}$ by $\frac{\varepsilon}{f_{l+1}}$ affects $W(t, s)$ if $v_{t}<h_{l+1}^{*}$.

Therefore the change in the objective function $\Delta Z_{\lambda}\left(s^{*}\right)$ is:

$$
\Delta Z_{\lambda}\left(s^{*}\right) \leq \varepsilon\left[\sum\left\{f_{t}: v_{t} \leq h_{s^{*}}^{*}\right\}\right]-\varepsilon\left[\sum\left\{f_{t}: v_{t}<h_{l+1}^{*}\right\}\right] .
$$

Hence $\Delta Z_{\lambda}\left(s^{*}\right) \leq 0$, and we conclude that $\lambda^{\prime}$ is also an optimal solution to $\left(\mathrm{LP}_{\lambda}\right)$.

Computing the change in discrepancy from $\lambda^{*}$ to $\lambda^{\prime}$ we observe that $\left[v_{s^{*}-1}-h_{s^{*}}^{*}\right]$ will decrease by $\varepsilon / f_{s^{*}}$. The term $\max \left\{h_{s^{*}}^{*}-h_{s^{*}+1}^{*}, 0\right\}$ goes up 
by $\varepsilon / f_{s^{*}}$. The term $\max \left\{h_{l}^{*}-h_{l+1}^{*}, 0\right\}$ goes down by $\varepsilon / f_{l+1}$. The contribution to discrepancy from other terms is unchanged.

Notice that the discrepancy changes by $\varepsilon / f_{s^{*}}-\varepsilon / f_{s^{*}}-\varepsilon / f_{l+1}<0$, contradicting our choice of $\lambda^{*}$ as the one with the smallest discrepancy.

Lemma 4 There is an optimal solution $\left(W^{*}, \lambda^{*}\right)$ to $\left(L P_{\lambda}\right)$ such that for any $j \geq s^{*}$

$$
\left\{t: v_{t} \leq h_{j}^{*}\right\}=\left\{t: v_{t}<h_{j+1}^{*}\right\}
$$

\section{Proof}

Let $\left(W^{*}, \lambda^{*}\right)$ be the optimal solution to $\left(\mathrm{LP}_{\lambda}\right)$ identified in Lemma 3 . If the Lemma is false there is a $j \geq s^{*}$ such that $h_{j}^{*}<h_{j+1}^{*}$. We construct a contradiction by considering a new set of $\left\{\lambda_{i}^{\prime}\right\}_{i=0}^{m}$, such that

$$
\begin{aligned}
\lambda_{j}^{\prime} & =\lambda_{j}^{*}-\varepsilon, \\
\lambda_{i}^{\prime} & =\lambda_{i}^{*} \quad \forall i \neq j
\end{aligned}
$$

Denote $h_{i}^{\prime}=i-\frac{1-F(i)}{f_{i}}-\frac{\lambda_{i}^{\prime}}{f_{i}}+\frac{\lambda_{i-1}^{\prime}}{f_{i}}$ for all $i$. This change results in the following changes to the values of $\left\{h_{i}^{*}\right\}_{i=0}^{m}$ :

$$
\begin{aligned}
h_{j}^{\prime} & =h_{j}^{*}+\frac{\varepsilon}{f_{j}}, \\
h_{j+1}^{\prime} & =h_{j+1}^{*}-\frac{\varepsilon}{f_{j+1}}, \\
h_{i}^{\prime} & =h_{i}^{*} \quad \forall i \notin\{j, j+1\} .
\end{aligned}
$$

Denote the change in the $\left(\mathrm{LP}_{\lambda}\right)$ problem objective function from changing $\lambda^{*}$ to $\lambda^{\prime}$ by $\Delta Z_{\lambda}\left(s^{*}\right)$. Consider the pairs $(t, s)$, for which $W(t, s)$ are affected by this change.

For $\varepsilon>0$ sufficiently small, increasing $h_{j}^{*}$ by $\frac{\varepsilon}{f_{j}}$ affects $W(t, s)$ only if $v_{t} \leq h_{j}^{*}$. Similarly, the decrease of $h_{j+1}^{*}$ by $\frac{\varepsilon}{f_{j+1}}$ affects $W(t, s)$ if $v_{t}<h_{j+1}^{*}$.

Therefore the change in the objective function $\Delta Z_{\lambda}\left(s^{*}\right)$ is:

$$
\Delta Z_{\lambda}\left(s^{*}\right) \leq \varepsilon\left[\sum\left\{f_{t}: v_{t} \leq h_{j}^{*}\right\}\right]-\varepsilon\left[\sum\left\{f_{t}: v_{t}<h_{j+1}^{*}\right\}\right] .
$$

Since $h_{j}^{*}<h_{j+1}^{*}$ the set $\left\{t: v_{t} \leq h_{j}^{*}\right\}$ is a subset of $\left\{t: v_{t}<h_{j+1}^{*}\right\}$. If it is a strict subset this would mean $\Delta Z_{\lambda}\left(s^{*}\right)<0$ contradicting optimality of $\left(W^{*}, \lambda^{*}\right)$. 
Theorem 6 Let $\left(W^{*}, \lambda^{*}\right)$ be the optimal solution identified in Lemma 4. There is an optimal solution $\left(a^{*}, a_{b}^{*}\right)$ to $\left(P_{\lambda^{*}}\right)$ such that

1. $a^{*}(t, s) \leq a^{*}(t+1, s)$ for all $t$,

2. $a_{b}^{*}(t, s) \leq a_{b}^{*}(t, s+1)$ for all $s$, and

3. $a_{b}^{*}\left(t, s^{*}\right)=a_{b}^{*}\left(t, s^{*}+1\right)=\ldots=a_{b}^{*}(t, m)$.

\section{Proof}

Now

$$
\begin{array}{r}
Z_{\lambda^{*}}\left(s^{*}\right)=\max \sum_{t=1}^{m} \sum_{s=1}^{m} f_{t} v_{t} f_{s} a(t, s)+\sum_{s=1}^{s^{*}-1} \sum_{t=1}^{m} f_{s} v_{s} f_{t} a_{b}(t, s)+\sum_{s=s^{*}}^{m} \sum_{t=1}^{m} f_{s} h_{s}^{*} f_{t} a_{b}(t, s) \\
\text { s.t. } a(t, s)+a_{b}(t, s) \leq 1 \forall t, s \\
a(t, s), a_{b}(t, s) \geq 0 \forall s, t
\end{array}
$$

This decomposes into a collection of subproblems, one subproblem for each profile $(t, s)$ of types. When $s \leq s^{*}-1$ the subproblem is

$$
\begin{gathered}
g_{\lambda^{*}}(t, s)=\max v_{t} a(t, s)+v_{s} a_{b}(t, s) \\
\text { s.t. } a(t, s)+a_{b}(t, s) \leq 1 \\
a(t, s), a_{b}(t, s) \geq 0
\end{gathered}
$$

and when $s \geq s^{*}$ it is

$$
\begin{gathered}
g_{\lambda^{*}}(t, s)=\max v_{t} a(t, s)+h_{s}^{*} a_{b}(t, s) \\
\text { s.t. } a(t, s)+a_{b}(t, s) \leq 1 \\
a(t, s), a_{b}(t, s) \geq 0
\end{gathered}
$$

For the case when $s \leq s^{*}-1$ the optimal solution is to award the good to the agent with highest non-negative virtual value. We will break ties in favor of the unconstrained bidder. Specifically, when $s \leq s^{*}-1$ we have the following.

1. When $v_{t} \geq \max \left\{v_{s}, 0\right\} \Rightarrow a^{*}(t, s)=1$.

2. When $v_{s}>\max \left\{v_{t}, 0\right\} \Rightarrow a_{b}^{*}(t, s)=1$. 
When $s \geq s^{*}$ we have the following.

1. When $v_{t} \geq \max \left\{h_{s}^{*}, 0\right\} \Rightarrow a^{*}(t, s)=1$.

2. When $h_{s}^{*}>\max \left\{v_{t}, 0\right\} \Rightarrow a_{b}^{*}(t, s)=1$.

Monotonicity of the virtual values and the $h^{*}$ s (from Lemma 3) yield items 1 and 2 of the Theorem.

To prove item 3 , suppose it is false. Then there is a $j \geq s^{*}$ and $t$ such that

$$
a_{b}^{*}(j+1, t)=1 \neq 0=a_{b}^{*}(j, t) .
$$

This can happen only if $h_{j}^{*} \leq v_{t}<h_{j+1}^{*}$. However, this contradicts Lemma 4 , since $\left\{t: v_{t} \leq h_{j}^{*}\right\}=\left\{t: v_{t}<h_{j+1}^{*}\right\}$.

\subsection{Solving $\left[B O P T\left(s^{*}\right)\right]$}

The optimal solution to $\left(\mathrm{P}_{\lambda^{*}}\right)$ satisfies the omitted monotonicity constraints and is therefore an optimal solution to $\left[\operatorname{BOPT}\left(s^{*}\right)\right]$. In particular, Theorem 6 establishes there is an optimal solution to $\left[B O P T\left(s^{*}\right)\right]$ such that $a_{b}(t, s)=$ $a_{b}\left(t, s^{*}\right)$ for all $t$ and all $s \geq s^{*}$.

This observation allows us to rewrite $\left[B O P T\left(s^{*}\right)\right]$ as

$$
\begin{gathered}
Z\left(s^{*}\right)=\max \sum_{s, t}\left[f_{t} \mathcal{A}_{t} v_{t}+f_{s} \mathcal{A}_{s}^{b} v_{s}\right] \\
\text { s.t. } \mathcal{A}_{1}^{b} \leq \ldots \leq \mathcal{A}_{m}^{b} \\
\mathcal{A}_{1} \leq \ldots \leq \mathcal{A}_{m} \\
\mathcal{A}_{i}=\sum_{s} f_{s} a(i, s) \forall i \\
\mathcal{A}_{i}^{b}=\sum_{t} f_{t} a_{b}(t, i) \forall i \\
a_{b}\left(t, s^{*}\right)=\ldots=a_{b}(t, m) \forall t \\
a(t, s)+a_{b}(t, s) \leq 1 \forall t, s \\
a(t, s), a_{b}(t, s) \geq 0 \forall t, s
\end{gathered}
$$


We will solve this version of $\left[B O P T\left(s^{*}\right)\right]$ by removing the monotonicity constraints and arguing that the solution to the relaxed problem satsifies them.

$$
\begin{gathered}
F\left(s^{*}\right)=\max \sum_{s, t}\left[f_{t} \mathcal{A}_{t} v_{t}+f_{s} \mathcal{A}_{s}^{b} v_{s}\right] \\
\text { s.t. } \mathcal{A}_{i}=\sum_{s} f_{s} a(i, s) \forall i \\
\mathcal{A}_{i}^{b}=\sum_{t} f_{t} a_{b}(t, i) \forall i \\
a_{b}\left(t, s^{*}\right)=\ldots=a_{b}(t, m) \forall t \\
a(t, s)+a_{b}(t, s) \leq 1 \forall t, s \\
a(t, s), a_{b}(t, s) \geq 0 \forall t, s
\end{gathered}
$$

This last program is equivalent to

$$
\begin{gathered}
F\left(s^{*}\right)=\max \sum_{t=1}^{m} \sum_{s=1}^{m} f_{t} f_{s} a(t, s) v_{t}+\sum_{t=1}^{m} \sum_{s=1}^{m} f_{t} f_{s} a_{b}(t, s) v_{s} \\
\text { s.t. } a_{b}\left(t, s^{*}\right)=\ldots=a_{b}(t, m) \forall t \\
a(t, s)+a_{b}(t, s) \leq 1 \forall t, s \\
a(t, s), a_{b}(t, s) \geq 0 \forall t, s
\end{gathered}
$$

Eliminating the $a_{b}(t, s)$ variables for $s \geq s^{*}+1$ we can rewrite the program as

$$
\begin{gathered}
F\left(s^{*}\right)=\max \sum_{t=1}^{m} \sum_{s=1}^{m} f_{t} f_{s} a(t, s) v_{t}+\sum_{t=1}^{m} \sum_{s=1}^{s^{*}-1} f_{t} f_{s} a_{b}(t, s) v_{s}+\sum_{t=1}^{m} f_{t}\left[\sum_{j=s^{*}}^{m} f_{j} v_{j}\right] a_{b}\left(t, s^{*}\right) \\
\text { s.t. } a(t, s)+a_{b}(t, s) \leq 1 \forall t=1, \ldots, m \forall s=1, \ldots, s^{*} \\
a, a_{b} \geq 0
\end{gathered}
$$

Set $w_{s}=v_{s}$ for $s \leq s^{*}-1$ and $w_{s^{*}}=\frac{\sum_{j=s^{*}}^{m} f_{j} v_{j}}{\sum_{j=s^{*}}^{m} f_{j}}$. Monotonicity of the $v_{s}$ 's implies that the $w_{s}$ 's are monotone as well. Set $g_{j}=f_{j}$ for $j \leq s^{*}-1$ and $g_{s^{*}}=\sum_{j=s^{*}}^{m} f_{j}$. Then

$$
F\left(s^{*}\right)=\max \sum_{t=1}^{m} \sum_{s=1}^{s^{*}} f_{t} g_{s} a(t, s) v_{t}+\sum_{t=1}^{m} \sum_{s=1}^{s^{*}} f_{t} g_{s} a_{b}(t, s) w_{s}
$$




$$
\begin{gathered}
\text { s.t. } a(t, s)+a_{b}(t, s) \leq 1 \forall t=1, \ldots, m \forall s=1, \ldots, s^{*} \\
a(t, s), a_{b}(t, s) \geq 0 \forall t=1, \ldots, m \forall s=1, \ldots, s^{*}
\end{gathered}
$$

This decomposes into a collection of subproblems one for each profile $(t, s)$ :

$$
\begin{gathered}
\max v_{t} a(t, s)+w_{s} a_{b}(t, s) \\
\text { s.t. } a(t, s)+a_{b}(t, s) \leq 1 \\
a(t, s), a_{b}(t, s) \geq 0
\end{gathered}
$$

Therefore, if we know the threshold, $s^{*}$, the optimal mechanism could be described thus:

1. If $v_{t}>\max \left\{w_{s}, 0\right\}$ then $a(t, s)=1$ and $a_{b}(t, s)=0$.

2. If $w_{s} \geq \max \left\{v_{t}, 0\right\}$ then $a_{b}(t, s)=1$ and $a(t, s)=0$.

3. If $v_{t}, w_{s}<0$ set $a_{b}(t, s)=a(t, s)=0$.

Monotonicity of the $v$ 's and $w$ 's ensures that the omitted monotonicity constraints are satisfied.

To determine the payments we use the fact that

$$
p_{s}^{b}=\sum_{j=0}^{s-1}(j+1)\left(\mathcal{A}_{j+1}^{b}-\mathcal{A}_{j}^{b}\right)=s \mathcal{A}_{s}^{b}-\sum_{j=1}^{s-1} \mathcal{A}_{j}^{b}
$$

and

$$
p_{t}=\sum_{j=0}^{t-1}(j+1)\left(\mathcal{A}_{j+1}-\mathcal{A}_{j}\right)=t \mathcal{A}_{t}-\sum_{j=1}^{t-1} \mathcal{A}_{j}
$$

for all $s, t$.

Let $r_{f}$ be the smallest index such that $v_{r_{f}} \geq 0$ and $r\left(s^{*}\right)$ the smallest index less than $s^{*}$ such that $w_{r\left(s^{*}\right)} \geq 0$. Then $\mathcal{A}_{t}=0$ when $t<r_{f}$ and $\mathcal{A}_{t}=\sum\left\{f_{s}: w_{s}<v_{t}\right\}=\operatorname{Pr}\left(w_{s}<v_{t}\right)$ when $t \geq r_{f}$. Similarly, $\mathcal{A}_{s}^{b}=0$ when $s<r\left(s^{*}\right)$ and $\mathcal{A}_{s}^{b}=\sum\left\{f_{t}: v_{t} \leq w_{s}\right\}=\operatorname{Pr}\left(v_{t} \leq w_{s}\right)$. Therefore, $p_{s}^{b}=0$ for $s<r\left(s^{*}\right)$ and

$$
p_{s}^{b}=s \operatorname{Pr}\left(v_{t} \leq w_{s}\right)-\sum_{j=1}^{s-1} \operatorname{Pr}\left(v_{t} \leq w_{j}\right) \forall s \geq r\left(s^{*}\right) .
$$

Similarly, $p_{t}=0$ for $t<r_{f}$ and

$$
p_{t}=t \operatorname{Pr}\left(w_{s}<v_{t}\right)-\sum_{j=1}^{t-1} \operatorname{Pr}\left(w_{s}<v_{j}\right) \forall t \geq r_{f} .
$$




\subsubsection{Determining $s^{*}$}

A condition that any candidate for the threshold $s^{*}$ must satsify is that

$$
b \geq p_{s^{*}}^{b}=s^{*} \operatorname{Pr}\left(v_{t} \leq w_{s^{*}}\right)-\sum_{j=1}^{s^{*}-1} \operatorname{Pr}\left(v_{t} \leq w_{j}\right) .
$$

For $j \leq s^{*}-1$ we know that $w_{j}=v_{j}$. Therefore $s^{*}$ can be chosen to be the largest index $s$ such that

$$
s \operatorname{Pr}\left(v_{t} \leq \frac{\sum_{i=s}^{m} f_{i} v_{i}}{\sum_{i=s}^{m} f_{i}}\right)-\sum_{j=1}^{s-1} \operatorname{Pr}\left(v_{t} \leq v_{j}\right) \leq b .
$$

\subsection{A Description of the Optimal Mechanism}

The optimal mechanism can be described in a way that is very similar to the description of the optimal mechanism in Myerson (1981).

1. First determine the optimal threshold $s^{*}$.

2. Compute for each type $s$ of the constrained bidder a modified virtual value $w_{s}$ as follows. If $s \leq s^{*}-1$ set $w_{s}=v_{s}$ and if $s \geq s^{*}$ set $w_{s}=\frac{\sum_{j=s^{*}}^{m} f_{j} v_{j}}{\sum_{j=s^{*}}^{m} f_{j}}$.

3. Compute for each type $t$ of the unconstrained bidder its virtual value.

4. If the unconstrained types virtual value exceeds the constrained types modified virtual value and is non-negative award the good to the unconstrained bidder. If the modified virtual value of the constrained bidder is at least as large as the virtual value of the unconstrained virtual value and is non-negative, award the good to the constrained bidder.

For this reason we will call the mechanism the threshold mechanism.

\section{Private Budget Constraint}

We turn now to the case when the budget of the constrained bidder is private information as well. In our mechanism the constrained bidder must post a bond equal to their reported budget. This ensures that the constrained 
bidders payment cannot exceed their reported budget. In terms of incentive compatability this will mean that we can ignore the possibility that the constrained bidder will inflate her budget. This allows us to focus on the case of a constrained bidder with a large budget pretending to have a smaller budget.

We discuss the Bayesian incentive compatible case only. Our goal will be to reduce the problem to the one considered in section 3 of this paper. A similar analysis applies in the dominant strategy incentive compatible case.

Let $\mathcal{A}_{i b}$ be the expected allocation to the constrained agent when the constrained agent reports $(i, b)$. Her expected payment will be $p_{i b}$. The requirement that the constrained bidder must post a bond implies that the constrained bidder can only underreport her budget. Therefore we only have to consider downward incentive constraints with respect to the reported budget, i.e.

$$
i \mathcal{A}_{i b}-p_{i b} \geq i \mathcal{A}_{i b^{\prime}}-p_{i b^{\prime}} \forall b^{\prime}<b .(D I C)
$$

We argue that the optimal mechanism in the case of a private budget constraint under the bond requirement is implemented through a variation of the threshold mechanism derived in section 3 of this paper. First, the bidders post bonds, revealing their budget constraints,. Then the threshold mechanism is implemented based on the revealed information about $b$. To prove optimality of the procedure it suffices to show that it satisfies (DIC).

Theorem 7 The optimal mechanism when the constrained bidder must post a bond is:

$$
\mathcal{A}_{i b}=\mathcal{A}_{i}^{b}, p_{i b}=p_{i}^{b}
$$

\section{Proof}

It suffices to verify that (DIC) is satisfied. Let $s_{b}, s_{c}$ be the threshold associated with budgets $b$ and $c$ where $b>c$. It is easy to see that $s_{b} \geq s_{c}$. If the constrained type is $i$, denote by $w_{i}^{b}$ and $w_{i}^{c}$ the modified virtual values associated with budgets $b$ and $c$ respectively. We have three cases.

1. $i<s_{c}$

Since $w_{i}^{b}=w_{i}^{c}$,

$$
\mathcal{A}_{i b}=\mathcal{A}_{i}^{b}=\operatorname{Pr}\left(v_{t} \leq w_{i}^{b}\right)=\operatorname{Pr}\left(v_{t} \leq w_{i}^{c}\right)=\mathcal{A}_{i}^{c}=\mathcal{A}_{i c} .
$$

A similar argument shows that $p_{i b}=p_{i}^{b}=p_{i}^{c}=p_{i c}$. Therefore (DIC) holds. 
2. $s_{c} \leq i<s_{b}$

As the threshold mechanism is incentive compatible with respect to reports of the value $i \mathcal{A}_{i b}-p_{i b}=i \mathcal{A}_{i}^{b}-p_{i}^{b} \geq i \mathcal{A}_{s_{c}}^{b}-p_{s_{c}}^{b}$. Now

$$
i \mathcal{A}_{s_{c}}^{b}-p_{s_{c}}^{b}=i \operatorname{Pr}\left(v_{t} \leq w_{s_{c}}^{b}\right)-\left[s_{c} \operatorname{Pr}\left(v_{t} \leq w_{s_{c}}^{b}\right)-\sum_{j=1}^{s_{c}-1} \operatorname{Pr}\left(v_{r} \leq w_{j}^{b}\right)\right]
$$

Since $w_{j}^{b}=v_{j}$ for all $j<s_{b}$ and $w_{j}^{b}=w_{j}^{c}=v_{j}$ for all $j<s_{c}$ we have that

$$
i \mathcal{A}_{s_{c}}^{b}-p_{s_{c}}^{b}=i \operatorname{Pr}\left(v_{t} \leq v_{s_{c}}\right)-\left[s_{c} \operatorname{Pr}\left(v_{t} \leq v_{s_{c}}\right)-\sum_{j=1}^{s_{c}-1} \operatorname{Pr}\left(v_{t} \leq w_{j}^{c}\right)\right] .
$$

Since $v_{s_{c}} \leq w_{s_{c}}^{c}$, the term on the right hand side is

$$
\begin{aligned}
& i \operatorname{Pr}\left(v_{t} \leq v_{s_{c}}\right)+i \operatorname{Pr}\left(v_{s_{c}}<v_{t} \leq w_{s_{c}}^{c}\right)-\left[i \operatorname{Pr}\left(v_{s_{c}}<v_{t} \leq w_{s_{c}}^{c}\right)+s_{c} \operatorname{Pr}\left(v_{t} \leq v_{s_{c}}\right)-\sum_{j=1}^{s_{c}-1} \operatorname{Pr}\left(v_{t} \leq w_{j}^{c}\right)\right] \\
& \geq i \operatorname{Pr}\left(v_{t} \leq w_{s_{c}}^{c}\right)-\left[i \operatorname{Pr}\left(v_{t} \leq v_{s_{c}}\right)-\sum_{j=1}^{s_{c}-1} \operatorname{Pr}\left(v_{t} \leq w_{j}^{c}\right)\right]=i \mathcal{A}_{i}^{c}-p_{i}^{c}=i \mathcal{A}_{i c}-p_{i c} .
\end{aligned}
$$

3. $i \geq s_{b}$

Incentive compatibility in the valuations and monotonicity of $\mathcal{A}^{b}$ implies

$$
i \mathcal{A}_{i b}-p_{i b}=i \mathcal{A}_{s_{b}}^{b}-p_{s_{b}}^{b} \geq i \mathcal{A}_{s_{c}}^{b}-p_{s_{c}}^{b} .
$$

Now

$$
\begin{gathered}
i \mathcal{A}_{s_{c}}^{b}-p_{s_{c}}^{b}=i \operatorname{Pr}\left(v_{t} \leq v_{s_{c}}\right)-\left[i \operatorname{Pr}\left(v_{t} \leq v_{s_{c}}\right)-\sum_{j=1}^{s_{c}-1} \operatorname{Pr}\left(v_{t} \leq v_{j}\right)\right] \\
=i \operatorname{Pr}\left(v_{t} \leq w_{s_{c}}^{c}\right)-\left[i \operatorname{Pr}\left(v_{t} \leq w_{s_{c}}^{c}\right)-\sum_{j=1}^{s_{c}-1} \operatorname{Pr}\left(v_{t} \leq v_{j}\right)\right]=i \mathcal{A}_{i}^{c}-p_{i}^{c} .
\end{gathered}
$$




\section{Acknowledgements}

We thank Peter Eso, Peter Klibanoff, Rudolf Muller and Hongwei Gui for useful comments.

\section{References}

1. Che, Y.-K., and I. Gale, 1996, "Expected Revenue of All-Pay Auctions and First-Price Sealed-Bid Auctions with Budget Constraints," Economics Letters, 50, 373-379.

2. Che, Y.-K., and I. Gale, 2000, "The Optimal Mechanism for Selling to a Budget-Constrained Buyer," Journal of Economic Theory, 92, 198-233.

3. Gavious, A. B. Moldovanu and A. Sela, 2002, "Bid Costs and Endogenous Bid Caps," RAND Journal of Economics, vol. 33(4), pages 709-722

4. Harris, M. and A. Raviv (1981), "A Theory of Monopoly Pricing Schemes with Demand Uncertainty," American Economic Review, 71, pp. 347-65.

5. Laffont, J.-J., and J. Robert, 1996, "Optimal Auction with Financially Constrained Buyers," Economics Letters, 52, 181-186.

6. Maskin, E. S., 2000, "Auctions, Development, and Privatization: Efficient Auctions with Liquidity- Constrained Buyers," European Economic Review, 44, 667-681.

7. Myerson, R., 1981, "Optimal Auction Design," Mathematics of Operations Research, 6, 58-73.

8. Zheng, C. Z., 2001, "High Bids and Broke Winners," Journal of Economic Theory, 100, 129-171. 